\title{
Evolution of beak morphology in the Ground Tit revealed by comparative transcriptomics
}

\author{
Yalin Cheng ${ }^{1,4 \dagger}$, Bin Gao ${ }^{1 \dagger}$, Haitao Wang ${ }^{2 \dagger}$, Naijian Han ${ }^{1}$, Shimiao Shao ${ }^{1,4}$, Shaoyuan Wu ${ }^{3}$, Gang Song ${ }^{1}$, \\ Yong E. Zhang ${ }^{1}$, Xiaojia Zhu ${ }^{1,4}$, Xin Lu ${ }^{5}$, Yanhua Qu ${ }^{1 *}$ and Fumin Lei ${ }^{14^{*}}$
}

\begin{abstract}
Background: Beak morphology exhibits considerable adaptive plasticity in birds, which results in highly varied or specialized forms in response to variations in ecology and life history. As the only parid species endemic to the Qinghai-Tibet Plateau, the Ground Tit (Parus humilis) has evolved a distinctly long and curved beak from other parids. An integration of morphometrics, phylogenetics, transcriptomics and embryology allows us to address the evolutionary and developmental mechanisms of the adaptive beak structure observed in the Ground Tit.

Results: A morphometric approach quantified that the Ground Tit has a comparatively longer and more decurved upper beaks than other parids. We estimated that the ancestor of the Ground Tit likely had a short straight upper beak similar to most current recognized parid species using an ancestral state reconstruction. This morphological specialization is considered an adaptation to its ground-oriented behavior on the high plateau. To identify genetic mechanisms behind this adaptive change, a comparative transcriptomic analysis was applied between the Ground Tit and its closely related species, the Great Tit (Parus major). We detected that 623 genes were significantly differentially expressed in embryonic upper beaks between the two species, 17 of which were functionally annotated to correlate with bone development and morphogenesis, although genes related to bone development were not found to undergo accelerated evolution in the Ground Tit. RT-qPCR validation confirmed differential expression of five out of eight genes that were selected from the 17 genes. Subsequent functional assays in chicken embryos demonstrated that two of these genes, FGF13 and ITGB3, may affect beak morphology by modulating levels of osteoblasts and osteoclasts.
\end{abstract}

Conclusions: Our results provide preliminary evidence that development of the long decurved beak of the Ground Tit is likely regulated by transcriptional activities of multiple genes coordinating osteoblasts and osteoclasts. The integration of multiple approaches employed here sheds light on ecological and genetic mechanisms in the evolution of avian morphology.

Keywords: Parus humilis, Beak, Morphology, Transcriptomics, Osteoblasts, Osteoclasts

\section{Background}

The avian beak is a highly evolvable structure. The malleability of beak morphology permits birds to adapt, sometimes rapidly, to diverse ecological niches [1]. Various interrelated ecological and behavioral aspects of diet and feeding, such as local and seasonal food availability, dietary

\footnotetext{
* Correspondence: quyh@ioz.ac.cn; leifm@ioz.ac.cn

${ }^{\dagger}$ Equal contributors

${ }^{1}$ Key Laboratory of Zoological Systematics and Evolution, Institute of

Zoology, Chinese Academy of Sciences, Beijing 100101, China

Full list of author information is available at the end of the article
}

preference, and acquisition and manipulation of food and water (e.g., food crushing, grasping, drinking and probing), likely act as the major selective forces on beak morphology [2]. Other factors in addition to diet, such as sexual selection and thermal regulation, may also play a role in shaping variation in beak morphology [3, 4]. Understanding the underlying genetic mechanisms involved in beak development is important for understanding the ultimate evolutionary causes of beak diversity in birds and the nature of evolutionary adaptation [5]. 
Studies of the genetic mechanisms underlying variation in beak morphology have identified multiple genes responsible for the development of beak morphology in birds. For example, BMP4 has been found to play a role in regulating both the width and depth of the prenasal cartilage (pnc) in the frontal nasal mass (FNM) of both Darwin's finches and chickens [6, 7]. The differential expression of other genes, such as CALM1,IHH, DKK3, $T G F B R 2$ and CTNNB1, is responsible for regulating the length and width of the pnc and premaxillary bone $(\mathrm{pmx})$ in birds [8-10]. Two recent genomic analyses on Darwin's finches revealed that variations of $A L X 1$ and HMGA2 are associated with beak shape [11] and beak size [12], respectively. These results show that the development of beak morphology is regulated by a complex genetic system that involves multiple genes. It is very similar to the craniofacial development across vertebrates, which is controlled by the conserved and complicated multi-gene pathways [13-15].

Traditionally, the Ground Tit (Parus humilis) was considered to be a member of the family Corvidae based on its phenotypic similarities with ground jays of the genus Podoces (e.g., the long and curved beak) $[16,17]$. However, phylogenetic studies placed the Ground Tit within the family Paridae and supported that its jay-like decurved beak is homoplasious [18, 19]. The Ground Tit is the only parid species that lives in the high altitude steppes and meadows of the Qinghai-Tibet Plateau, where it resides between 3100 and $5500 \mathrm{~m}$. Other Paridae members are distributed in forested habitats in low altitudinal areas across Eurasia, North America and Africa [20]. In response to the selective pressure imposed by the foraging pattern and burrow-nesting habits in the sparsely vegetated, treeless and high altitude environment [21, 22], the Ground Tit has evolved a unique beak morphology that is distinct from the short and straight beak of all other parids [23].

To explore the evolutionary and developmental mechanisms of the adaptive beak structure observed in the Ground Tit, we firstly employed linear and geometric analyses to quantify variations in beak morphology between the Ground Tit and other parids. We then identified potential candidate genes contributing to beak development in the Ground Tit by comparative transcription between the Ground Tit and the Great Tit (Parus major). They are closely related species with a divergence time of approximately 7.7-9.9 million years [19]. We further tested the function of the candidate genes in chicken embryos with recombinant proteins. Our results shed new light on the evolution of beak morphology in a non-model species and possibly in other extensive bird species as well.

\section{Results}

\section{A derived long decurved beak in the Ground Tit}

Length, width and depth of upper beaks from 349 skin specimens were measured for linear analysis (Fig. 1a). There were no significant phylogenetic signals in three size characteristics (beak length, width, depth) of the parids (see Additional file 1: Table S1), suggesting that beak size evolved independently from phylogeny. Both Hotelling-Lawley's test (HotellingLawley trace $\left.=20.51, F_{36}, 998=189.54, P<0.001\right)$ and linear discriminant analysis (LDA) showed that beak size in the Ground Tit is significantly different from that in all other parids (Fig. 1b; see Additional file 1: Tables S2 and S3). However, the LDA without beak length could not discriminate Ground Tits from other parids (Fig. 1c; see Additional file 1: Tables S2 and S3), which indicated that beak length is a better discriminant variable than beak width and depth. An LDA with only beak length further showed that the length is the major variation in beak size between the Ground tit and other parids (see Additional file 1: Table S3, Figure S1a), which is similar to previous results based on a subsection of current dataset [23] (see Additional file 2).

A total of 352 upper beak profiles collected from our previous work [23] and newly photographed images were used to perform geometric analysis (Fig. 1a, see Additional file 3). We did not find significant phylogenetic signals in Procrustes coordinates (shape, $P=0.802$ ) and centroid size (size, $P=0.431$ ), indicating that evolution of beak shape was also phylogenetically independent. Multivariate regression detected no significant allometry between shape and size $(P=0.064)$ even though $49.04 \%$ of total interspecific variation in shape was predicted by size variation. Procrustes ANOVA on Procrustes coordinates showed significant interspecific difference in beak shape $\left(F_{2808}, 79,326=63.14, P<0.001\right)$. Discriminant function analysis (DFA) and canonical variate analysis (CVA) clearly distinguished Ground Tits from all other parids (Fig. 1d, see Additional file 1: Tables S4 and S5). Although CV1 explained only 35.70\% of total variation in beak shape across 13 species (see Additional file 1: Table S6), the variation in CV1 represented differences between the Ground Tit and other parids (Fig. 1d, see Additional file 1: Figure S1b), which was indicated by the change of beak shape from a blunt, robust and straight to a pointed, slender and decurved one in CV1 axis (Fig. 1d).

An ancestral state reconstruction of beak morphology estimated that both the ancestor of Ground Tits and the common ancestor of the parids had beak morphology similar to that of the majority of extant taxa within the Paridae (Fig. 2). The ancestral beak of Ground Tits was medium sized and straight shaped, resulting in a short 
a

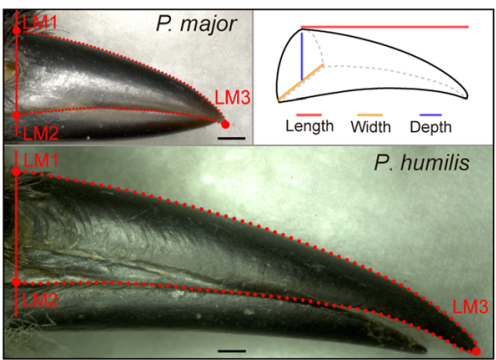

C

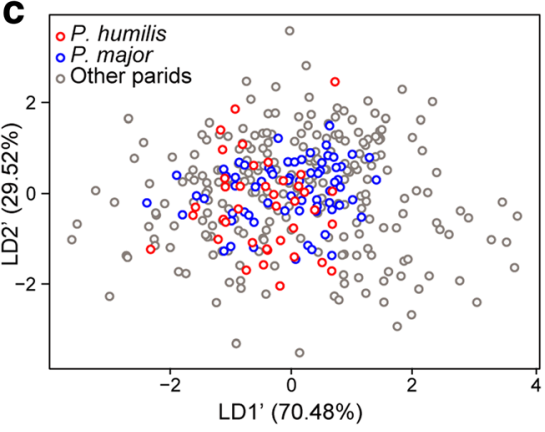

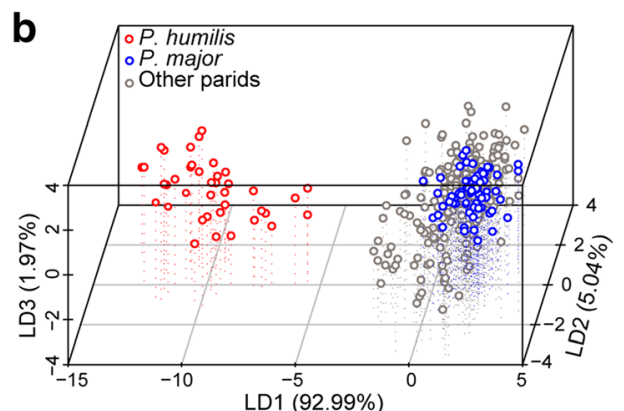

d

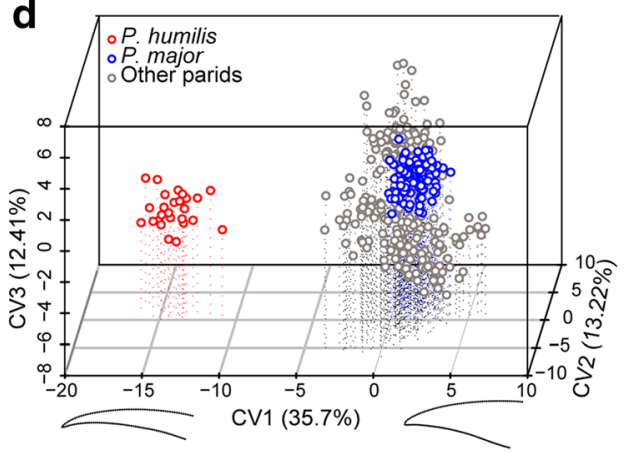

Fig. 1 Morphometric analyses for beak size and beak shape of 13 parid species. a. The rule of measurement for the size (length, width and depth) of upper beaks and the selection of landmarks and semi-landmarks on upper beaks. Scale bars are equal to $1 \mathrm{~mm}$. b. LDA for all three size parameters. The 3D plot is based on three linear discriminant functions: LD1, LD2 and LD3. Ground Tits are clearly separated from other tits. c. LDA for beak width and depth. Ground Tits overlap with other parids. d. CVA of beak shape based on Procrustes coordinates. The first three CV components, CV1, CV2 and CV3, cumulatively account for 61.33\% of total shape variation. Ground Tits are clearly separated from other parids. CV1 predicted the variations in beak shape between the Ground Tit and all other parid species. CV2 and CV3 predicted differences among other parids (see Additional file 1: Figure S1b)
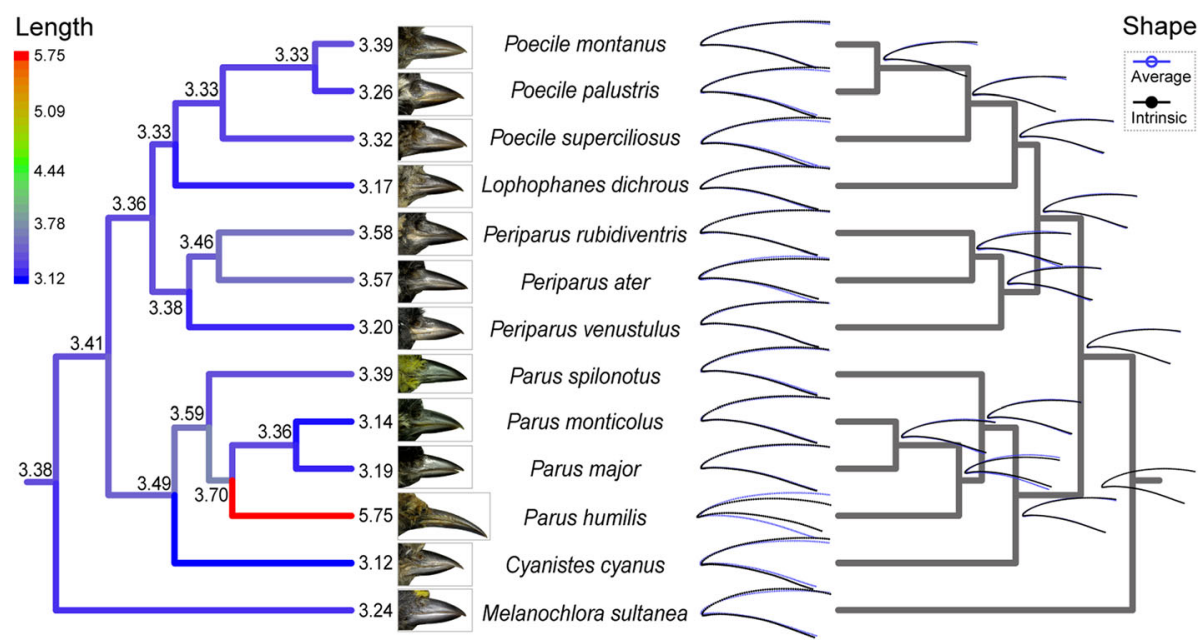

Fig. 2 Ancestral states reconstruction for beak length and shape of 13 parid species. The node labels in the length tree (left) are the estimated length of ancestral beaks. The beak profiles corresponding to the nodes in the shape tree (right) are the estimated ancestral beak shapes. The blue profiles are the average shape, while the black profiles are the real shape. The images of beaks were taken from specimen of National Zoological Museum of the Institute of Zoology, Chinese Academy of Sciences 
and relatively straight beak compared to extant Ground Tits (Fig. 2; see Additional file 1: Figure S2). Similarly, the putative common ancestor of the parids exhibited a medium sized and straight shaped beak.

\section{Coding sequence in beak-related genes underwent unaccelerated evolution}

A total of 6310 orthologous genes among the Ground Tit, Great Tit, Medium Ground Finch (Geospiza fortis), Atlantic Canary (Serinus canaria) and American Crow (Corvus brachyrhynchos) were identified for phylogenomic analysis. After removing genes with errors and saturated mutations, 1873 genes were retained for subsequent analyses. For all 1873 genes, the mean $\mathrm{dN} / \mathrm{dS}$ ratio of the Ground Tit was higher than that of other lineages, but these differences were not statistically significant (Fig. 3a; see Additional file 1: Tables S7 and S8). And the genes with $\mathrm{dN} / \mathrm{dS}>1$ in the Ground Tit were found to participate in repairing damaged DNA and responding to stress instead of dedicating to bone morphogenesis (see Additional file 1: Table S9).

When we functionally annotated the 1873 retained genes, 49 genes that were identified to contribute to bone development and remodeling (see Additional file 1: Table S10) did not show statistically significant differences in $\mathrm{dN} / \mathrm{dS}$ ratios between the Ground Tit and the other four lineages (Fig. 3a; see Additional file 1: Tables S7 and S8), although they have quite different beak morphotypes. Moreover, none of the 49 genes in the Ground Tit was assigned both an elevated $\mathrm{dN} / \mathrm{dS}$ ratio and a significant likelihood value (see Additional file 1: Figure S3).

\section{Gene expression differentiation between two beak morph} types of the Ground Tit and the Great Tit

Transcriptomes were sequenced for four embryonic upper beaks at stages 28/29 (see Additional file 1: Figure S4) from two Ground Tits and two Great Tits (see Additional file 1: Figure S5). Approximately 19.53-19.99 and 22.88-25.30 million clean reads were mapped to the Ground Tit [19] and the Great Tit [24] genomes, respectively (see Additional file 1: Figure S6a). Expressions of 9217 orthologous genes displayed considerably higher variation between species than that within species (see Additional file 1: Figure S6b). DESeq identified 2730 genes with statistically significant differences in expression between Ground Tits and Great Tits, edgeR identified 3183 significantly different genes, and NOISeq identified 1262 (see Additional file 1: Figure S7a-c). By combining results of above three approaches (see Methods), we defined 623 genes to be significantly differentially expressed, including 148 up-regulated and 475 down-regulated genes in the Ground Tit relative to the
Great Tit (see Additional file 1: Figure S7d; see Additional file 4).

Although KEGG enrichment analysis of differentially expressed genes did not identify pathways with significantly FDR-adjusted $P$-values, we found several conserved pathways to be likely relevant to beak or face morphogenesis [25], including Wnt (ko04310), MAPK/ FGF (ko04010), Osteoclast differentiation (OCD) (ko04380), Calcium (ko04020) and Notch signaling pathways (ko04330) (see Additional file 1: Table S11, Figure S8a). These pathways involved 26 genes, 17 of which were functionally annotated to be related to bone development and morphogenesis, including 3 upregulated and 14 down-regulated genes (Table 1 and Fig. 3b, see Additional file 1: Table S12). The function categories of these genes were mostly clustered in the top $20 \mathrm{GO}$ terms, such as cartilage development (GO:0051216), anatomical structure morphogenesis (GO:0009653), connective tissue development (GO:0061448) and skeletal system development (GO:0001501) (see Additional file 1: Figure S8b).

Eight genes were selected from the 17 genes (see Methods) to be validated for their observed expression levels and patterns in embryonic upper beaks at stages 28/29 using reverse transcription quantitative PCR (RTqPCR). RT-qPCR validation confirmed five out of the eight genes showing significant differences in expression in the upper beaks of Ground Tits relative to that in Great Tits, including FGF13 in FGF signaling pathway, $F R Z B$ and WIF1 in canonical Wnt signaling pathway, and ITGB3 and NFATC1 in OCD signaling pathway (Table 1 and Fig. 3c). Spatial expressions of three genes with the highest fold-changes (one from each pathway: WIF1, FGF13 and ITGB3) were assayed by in situ hybridization on sections of upper beaks at stages HH28/29. In situ hybridization supported transcriptome and RT-qPCR results that Ground Tits expressed higher level of FGF13 but lower levels of WIF1 and ITGB3 than Great Tits (Fig. 3d). Their expressions were localized to the upper beak processes in both species.

\section{Gain of FGF13 and ITGB3 proteins affected beak morphology in chicken embryos}

Chicken embryos treated with recombinant FGF13 protein (rFGF13) and recombinant ITGB3 protein (rITGB3) showed an elevated proportion between upper and lower beak length relative to control embryos (Fig. 4a-d). These increases in ratios were statistically significant (Fig. 4f). However, we did not find significant decreases in ratios of upper to lower beak length in rWIF1-treated embryos compared to controls (Fig. 4e, f). Consistently, we also observed apparently increased amounts of osteoblasts in rFGF13-treated embryos but not in rWIF1treated ones (Fig. 4g-i), and obvious reductions in 


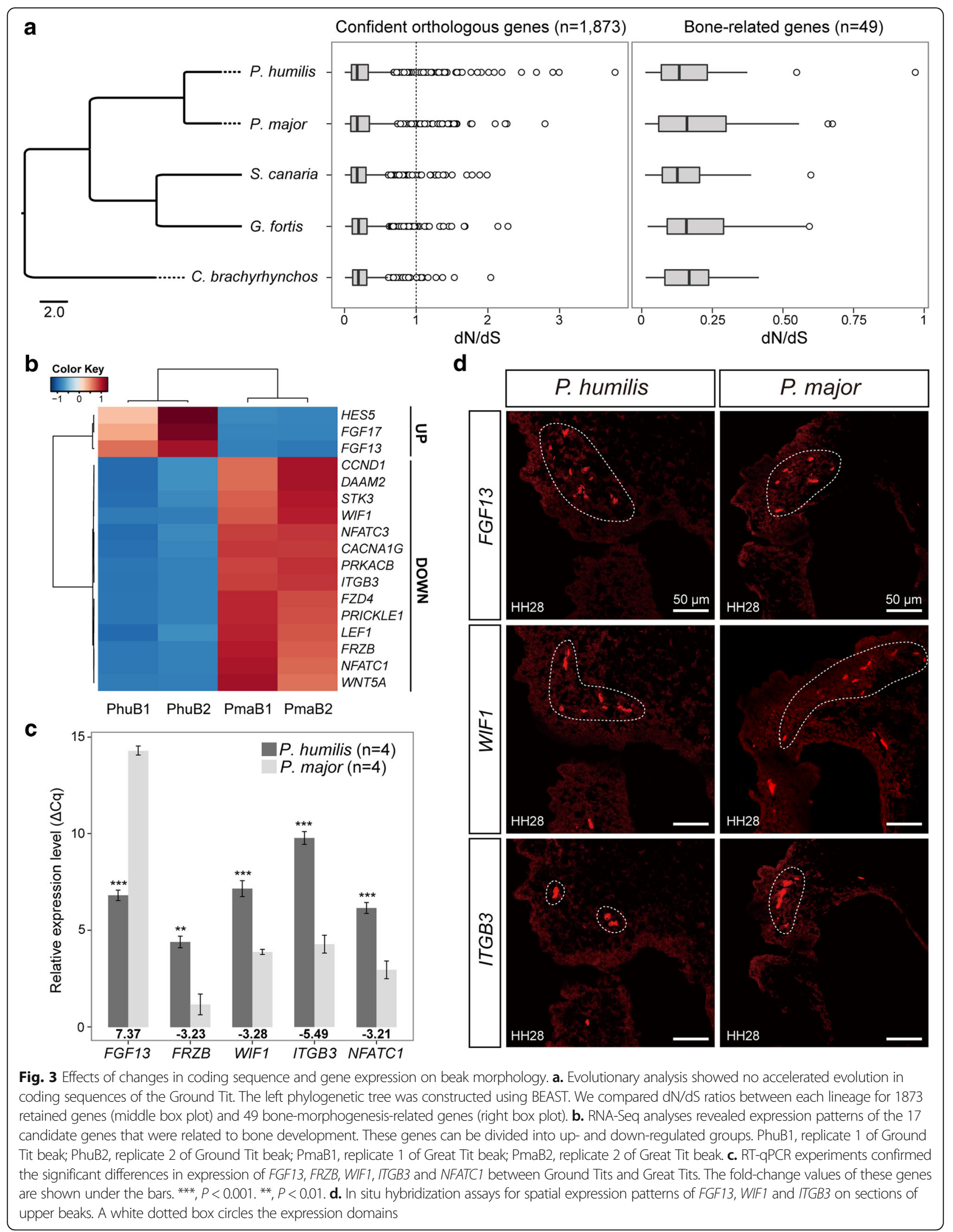


Table 1 Descriptions and expression patterns of 17 differentially expressed genes that probably correlate with beak morphology

\begin{tabular}{|c|c|c|c|c|c|c|c|c|}
\hline \multirow[t]{2}{*}{ Pathways } & \multirow[t]{2}{*}{ Genes } & \multirow[t]{2}{*}{ Descriptions } & \multicolumn{2}{|l|}{ TPM } & \multicolumn{2}{|l|}{ NOISeq } & \multicolumn{2}{|l|}{ RT-qPCR } \\
\hline & & & P. humilis & P. major & $\log 2 \mathrm{FC}$ & $P$-adj & $\log 2 \mathrm{FC}$ & $P$ \\
\hline \multirow[t]{10}{*}{ Wnt } & WNT5A & Protein Wnt-5a & 48.06 & 148.61 & -1.63 & $0.00 \mathrm{E}+00$ & - & - \\
\hline & PRICKLE1 & Prickle-like protein 1 & 14.86 & 55.20 & -1.89 & $2.75 \mathrm{E}-06$ & -0.17 & 1.10E-01 \\
\hline & $W \mid F 1^{*} \dagger$ & Wnt inhibitory factor 1 & 7.28 & 57.05 & -2.97 & 8.87E-06 & -3.28 & $5.30 \mathrm{E}-06$ \\
\hline & NFATC3 & Nuclear factor of activated T-cells, cytoplasmic 3 & 8.98 & 39.30 & -2.13 & 9.47E-05 & - & - \\
\hline & FZD4 & Frizzled-4 & 16.67 & 89.95 & -2.43 & $1.54 \mathrm{E}-04$ & - & - \\
\hline & LEF1 & Lymphoid enhancer-binding factor 1 & 60.50 & 143.01 & -1.24 & 1.77E-04 & - & - \\
\hline & $F R Z B^{*}$ & Secreted frizzled-related protein 3 & 71.32 & 150.58 & -1.08 & 3.47E-04 & -3.23 & 4.12E-05 \\
\hline & DAAM2 & Disheveled-associated activator of morphogenesis 2 & 2.48 & 15.04 & -2.60 & 3.47E-04 & - & - \\
\hline & CCND1 & G1/S-specific cyclin-D1 & 24.35 & 69.13 & -1.51 & 4.18E-04 & - & - \\
\hline & PRKACB & CAMP-dependent protein kinase catalytic subunit beta & 34.22 & 73.42 & -1.10 & 4.97E-04 & - & - \\
\hline \multirow[t]{3}{*}{ MAPK/FGF } & FGF17 & Fibroblast growth factor 17 & 45.91 & 0.38 & 6.91 & $0.00 E+00$ & 0.50 & $4.18 \mathrm{E}-01$ \\
\hline & FGF13* ${ }^{*}$ & Fibroblast growth factor 13 & 11.33 & 3.71 & 1.61 & 4.21E-04 & 7.37 & $1.23 \mathrm{E}-08$ \\
\hline & STK3 & Serine/threonine-protein kinase 38 & 11.70 & 40.72 & -1.80 & 4.64E-04 & - & - \\
\hline \multirow[t]{2}{*}{ OCD } & NFATC1* & Nuclear factor of activated T-cells, cytoplasmic 1 & 5.82 & 39.32 & -2.76 & $1.60 \mathrm{E}-08$ & -3.21 & $2.16 \mathrm{E}-05$ \\
\hline & $I T G B 3^{*}+$ & Integrin beta-3 & 0.76 & 6.43 & -3.08 & 8.57E-05 & -5.49 & $1.21 \mathrm{E}-06$ \\
\hline Notch & HES5 & Transcription factor HES-5 & 117.76 & 17.23 & 2.77 & $3.11 \mathrm{E}-04$ & 0.22 & $6.83 \mathrm{E}-01$ \\
\hline Calcium & CACNAIG & Voltage-dependent T-type calcium channel subunit alpha-1G & 4.28 & 14.60 & -1.77 & 5.79E-04 & - & - \\
\hline
\end{tabular}

RNA-Seq analyzed expression patterns that were subsequently validated by RT-qPCR, in situ hybridization and functional experiments. The expression levels and patterns are shown with the results of NOISeq. Please see results of DESeq and edgeR in Additional file 2: Table S11. TPM (transcript per million) represents the expression level. Log2FC is the transformation of the fold change in expression levels. $P$-adj is the adjusted $P$-value using FDR, which is considered equivalent to $1-\mathrm{q}_{\mathrm{NO}}$ seq in NOISeq. $P$ in the last column is produced by student's t-test for the relative expression of both species from RT-qPCR. Nine non-validated genes in RT-qPCR are filled with "-" in last two columns. Five confirmed genes by RT-qPCR are marked with "*". " $\dagger$ " marks the three genes assayed by in situ hybridization. Two proved genes by functional experiments are bolded

amounts of osteoclasts in both rFGF13- and rITGB3treated embryos (Fig. $4 \mathrm{j}-1)$.

\section{Discussion}

\section{Evolutionary dynamics of the specialized beak in the Ground Tit}

Geometric analysis separated more species pairs and detected differences among all species (see Additional file 1: Tables S4 and S5), while linear analysis only distinguished the Ground Tit from other parids (see Additional file 1: Tables S3), showing that the geometric analysis may be better to discriminate interspecific variations of parids than the linear analysis. Both morphometric analyses, however, could clearly separate the Ground Tit from all other parids mainly based on length and curvature variables. Although the curvature and length of the beak had no significant allometry, nearly half variation in curvature could be predicted by the variation of length, suggesting that beak curvature was co-evolved with beak length to some extent rather than completely independent. Ancestral reconstruction estimated that the long and decurved beak of the Ground Tit evolved from a short and straight ancestral type that is retained in most other extant parids. This morphological change in Ground Tits is likely to be an adaptation to a ground-living lifestyle in alpine steppe and meadow habitats created by the uplift of the Qinghai-Tibet Plateau [26].

Variation in beak morphology coincides with variation in diet and feeding behaviors in birds [1]. Ground Tits feed mostly on soil arthropods, such as insects and larvae, and occasionally on grass seeds and roots [22]. In sparse plateau environments with limited food resources [26], it is important for Ground Tits to be able to probe the turf and soil for food. A long and decurved beak is better suited for this terrestrial feeding behavior compared to the short and straight beaks of other parids, which occupy on arboreal niches.

The evolution of a long and decurved beak is also beneficial to Ground Tits for the construction of terrestrial nests in an open environment. Ground Tits nest in excavated burrows in ditches, pits, vertical banks or slopes [21]. Based on our field observations, Ground Tits excavate nests in banks or slopes by first using their beak and then using their talons to remove the soil. This burrowing behavior of Ground Tits probably also acted as a selective force in the development of their long and decurved beak. 

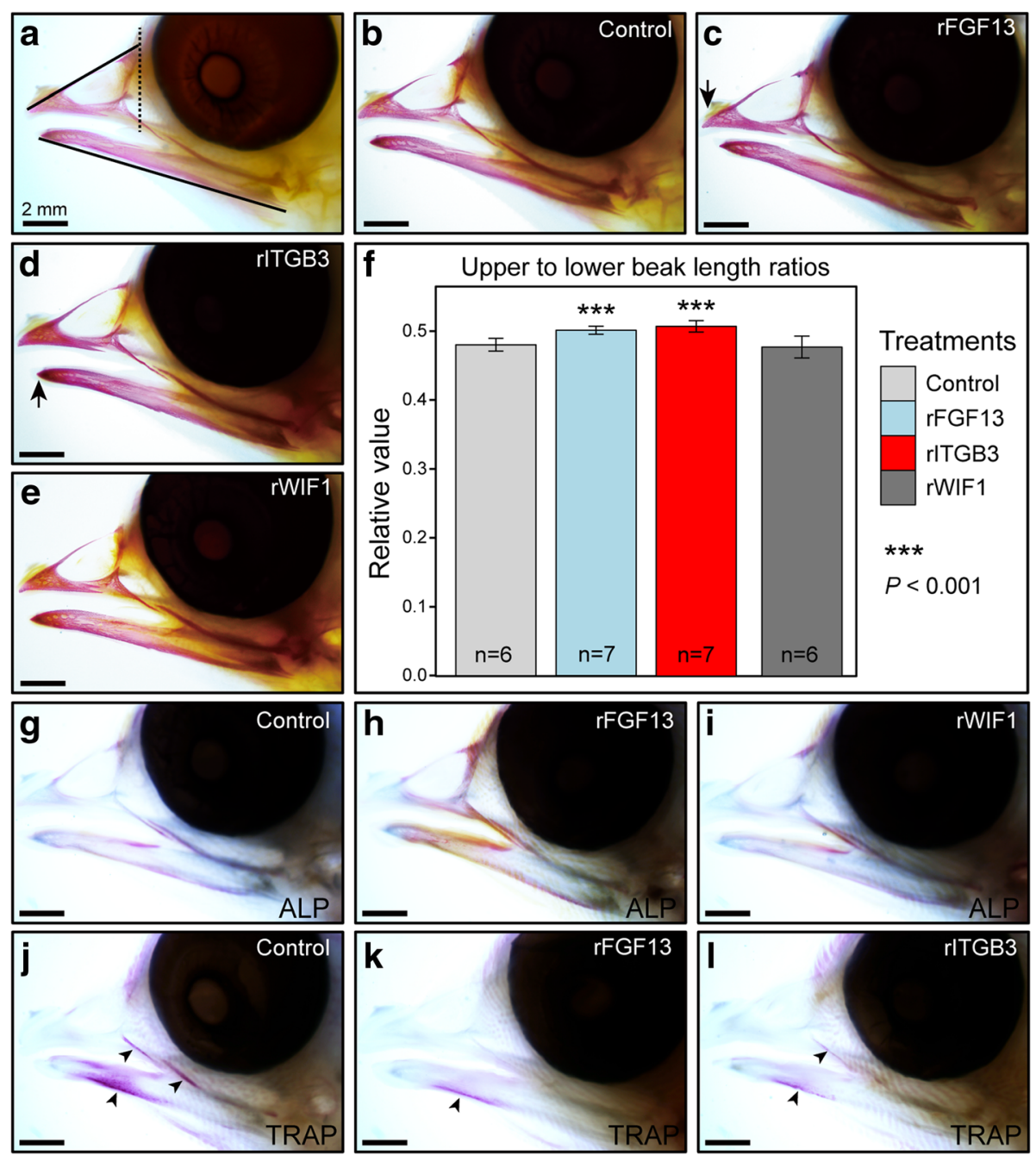

Fig. 4 Injections of recombinant proteins affected beak development in chicken embryos. All embryos were injected at HH30 and were collected at $\mathrm{HH} 38 . \mathrm{HH}$, Hamburger and Hamilton stage. Bones of embryonic heads at HH38 were stained by alizarin red (a-e). a. The rule of measurements for the length of the upper and lower beak (arrow). b. Control embryos were treated with BSA. c. rFGF13-treated embryos had longer beaks. d. rITGB3 shortened the beak, especially the lower beak (arrow). e. No differences were observed in rWIF1-treated embryos. f. Quantification of treatment-dependent changes in length relative to control embryos. g. Whole mount ALP staining in the chicken head detected osteoblasts (stained purple) around the upper and lower beak skeleton (control). h. More robust ALP-positive staining in rFGF13-treated embryos $(n=3)$ demonstrated higher density of osteoblasts than control $(n$ =4). i. Levels of osteoblasts in rWIF1-treated embryos $(n=2)$ did not change. j. Whole mount TRAP staining detected more osteoclasts (stained purple) in the lower beak than the upper (arrowhead). $\mathbf{k}$-I. Less TRAP-positive staining in rFGF13-treated $(n=2)$ and rITGB3-treated embryos $(n=3)$ demonstrated lower density of osteoclasts compared to control $(n=3)$

\section{Changes in gene expression regulating beak development}

Increasing researches have demonstrated that regulation of gene expression rather than functional coding change drives adaptively phenotypic evolution [5, 27], including some studies on evolution of beak morphology in birds [6-10]. Our results also showed that genes related to beak formation and development were not under selective constraints in terms of functional coding differences, but some of them were significantly differential expressed in embryonic beaks between Ground Tits and Great Tits, suggesting a transcriptional regulation in beak development. However, several other known genes, which have been discovered in contribution to beak morphogenesis of other birds $[6-9,28-30]$, were not found to be expressed differently between Ground Tits and Great Tits (see Additional file 1: Table S13). This inconsistency reflects that molecular programs of beak patterning are highly species-specific, which is a complex but flexible process involving multiple genes, signaling pathways, and reciprocal signaling interactions. Applications of different analyzing programs might also introduce the discordance by their own data-processing and statistical methods [31]. For example, DESeq and 
edgeR used in the current study found differential expression of RALDH2 but NOISeq did not. In this case, DESeq and edgeR identified over 2000 differentially expressed genes that were not identified by NOISeq, which may result from poor FDR control for small sample sizes [31]. To improve true positives, we combined three approaches and conducted further validation.

RT-qPCR validation confirmed expression levels and patterns of five candidates, two of which, FGF13 and ITGB3, were functionally proved in chicken embryos. FGF13 is a member of the FGF signaling family (FGFs) in the FGF pathway. Previous reports have highlighted roles of FGFs in inducing neural crest cells to promote skeletal outgrowth of the beak [28, 29, 32, 33]. In our results, the injection of rFGF13 elevated the proportion between upper and lower beak length of chicken embryos and increased amounts of osteoblasts relative to control. We assumed that FGF13 might elongate the upper beak of embryos by increasing osteoblast formation, because FGFs have been involved in the generation of osteoblasts [34] that regulate bone deposition in the face and beak during embryonic development $[35,36]$. However, we possibly underestimated the actual elongation of the beak by simply comparing ratios of upper to lower beaks, because rFGF13 increased osteoblasts in both upper and lower beaks of chicken embryos (Fig. 4h).

ITGB3, an adhesion receptor highly expressed in osteoclasts, is required for the interaction of osteoclasts with bone substrates during bone resorption [37, 38]. ITGB3blocked mice have been found to stack more osteoclasts that are unable to adhere to bone substrates, and thus display a defect in bone resorption [37, 39]. Similarly, our functional experiments showed that rITGB3-treated chicken embryos held less osteoclasts relative to controls. We suggested that increases of ITGB3 protein probably boosted the adhesion of osteoclasts to bones, which drove embryos to consume more osteoclasts to participate in bone resorption. Osteoclast-dominated bone resorption has been discovered to negatively affect beak length during development [36]. Consequently, elevated proportions between upper and lower beak in rITGB3-treated embryos could presumably be results of the shortening of lower beaks, because more osteoclasts in lower beaks of chicken embryos could be used to take part in bone resorption than that in upper beaks (Fig. 4j). Moreover, we also observed reduced osteoclasts in rFGF13-treated embryos, reinforcing the negative regulation of osteoblasts to osteoclasts [40].

Taken together, our functional experiments preliminarily demonstrated the roles of the candidate genes, identified by RNA-Seq, in beak morphogenesis; FGF13 and ITGB3 might affect the beak morphology by regulating levels of osteoblasts and osteoclasts, respectively.
Although FGF13 and ITGB3 were found to affect both upper and lower beak parts in chicken embryos, we infer that they could affect the upper beak more than the lower beak in embryos of Ground Tits and Great Tits according to their higher expression in upper beaks (Fig. 3d). In the context of the Ground Tit, therefore, we suggest that a long decurved upper beak could be most likely regulated by FGF13- and ITGB3-mediated osteoblasts and osteoclasts.

\section{Conclusion}

Our results show that the ground-oriented Ground Tit evolved a comparatively longer and more decurved beak from a short straight form which is similar to most other Paridae species. The adaptive variation of the beak morphology is likely associated with changes in gene expression rather than mutations in coding sequences. Our functional assays also show that changing transcriptional activities of genes may affect beak morphology by modulating osteoblasts and osteoclasts.

\section{Methods \\ Morphometric analyses}

We examined 349 study skin specimens from 13 Paridae species with body mass information recorded in the National Zoological Museum of the Institute of Zoology, Chinese Academy of Sciences. We used a digital caliper to measure the length (from the rostral edge of the nares to the tip), depth (at the nares) and width (at the nares) of the upper beak (Fig. 1a) that determines the speciesspecific morphology of the avian beak [41] and reflects the functional biomechanical properties of the entire beak [42, 43]. Beak length data of 282 specimens were from our previous research [23]. All measurements were standardized by the cube root of body mass to account for body size dependence [44] (see Additional file 1: Table S14; see Additional file 2). To test phylogenetic independence for linear measurements (length, depth and width), we used Blomberg's K [45] and Pagel's $\lambda$ [46] to assess their phylogenetic signals under a Brownian motion (BM) model based on a published phylogenetic tree of parids [47]. For both indices, a value close to zero indicates phylogenetic independence and a value of one indicates that traits are evolving under BM. Subsequently, we evaluated the overall variation in linear measurements using Hotelling-Lawley's test and conducted LDAs to discriminate Ground Tits from other parids.

We also compared the shape of the upper beak in the Ground Tit with that in other parids using geometric morphometric analysis. A total of 352 lateral images of upper beaks of the skin specimens were used in the geometric analysis (see Additional file 3), including $292 \mathrm{im}$ ages collected from our recently published work [23] and 60 newly photographed images using a microscopic 
image collecting system. We placed 3 landmarks and 116 sliding semi-landmarks on each beak image (Fig. 1a) to characterize the shape of the upper beak using TPSDIG [48]. To eliminate the effect of location, direction and scale on beak shape, we used generalized least square superimposition to rotate, translate and scale landmark coordinates in TPSRELW [49]. We firstly tested phylogenetic signals on both Procrustes coordinate and centroid size with 10,000 random permutations [50] using Collect Statistics on Tree Set option in Morpho J [51]. To assess allometric relationship between beak shape and size, additionally, we performed a multivariate regression of Procrustes coordinates onto centroid size under Regression option [52]. Procrustes ANOVA and CVA were used to analyze interspecific variations of the beak shape [51]. The CVA is a method used to extract the axes with the greatest interspecific differences and generate a matrix of pairwise Mahalanobis distances [53]. DFA was performed to examine the separation between each species pair [54]. All specimens used are adults without significant variations in beak size and beak shape between males and females (see Additional file 1: Table S15). Non-parametric Wilcoxon rank sum test was used to examine the difference between sexes.

\section{Ancestral state reconstruction}

To estimate ancestral beak morphology of the Ground Tit and other parids, we performed an ancestral state reconstruction for beak morphology based on the phylogenetic tree of parids, including beak size and beak shape. The ancestral states of the beak size were inferred using a maximum likelihood method implemented in $\mathrm{R}$ using the APE package [55], while those of the beak shape were estimated based on Procrustes coordinates with map onto phylogeny option using squared-change parsimony method [56] in Morpho J.

\section{Orthology and evolutionary analyses}

Genomic coding sequences from five avian species, including the Ground Tit [19], Great Tit (NCBI, Parus_major1.1), Medium Ground Finch (NCBI, GeoFor_1.0), Atlantic Canary (NCBI, SCA1) and American Crow (NCBI, ASM69197v1), were used in this analysis. The longest transcript isoform was retained for each gene, and genes shorter than $150 \mathrm{bp}$ were discarded. Putative orthologous among above five species were identified using an all-against-all BLAST with an E value 1E-10. Each orthologues gene was aligned and trimmed. Phylogeny and divergence time were estimated using a substitution rate of $\sim 3.3 \times 10^{-3}$ substitutions per site per million years [57] in BEAST 2 [58]. Based on this phylogeny, we estimated lineage-specific evolutionary information, such as $d N, d S$ and $d N / d S$, using Codeml program in PAML package under the free-ratio model [59]. To reduce errors and avoid saturated mutations, we retained only the genes with $\mathrm{N}^{*} \mathrm{dN}>1, \mathrm{~S}^{*} \mathrm{~d} S>1$ and $\mathrm{d} S<1$ [60]. The likelihood values between the alternative and null models were compared to test differences in $\mathrm{dN} / \mathrm{dS}$ ratios among lineages [61]. $P$-values were adjusted for multiple tests with false discovery rate (FDR) [62]. FDR-adjusted $P$-values below 0.05 were used as the threshold for statistical significance. Wilcoxon rank sum tests were used to test whether the mean $\mathrm{dN} / \mathrm{dS}$ value of the Ground Tit differs significantly from those of other lineages.

\section{Selection and identification of developmental stages}

At early stages of embryonic development, initiation, migration and differentiation of neural crest cells contribute to avian beak morphogenesis [63, 64]. Beak morphogenesis involves multiple facial prominences, including FNM, lateral nasal prominence (LNP), maxillary prominence (MXP) and mandibular prominence (MDP) [65]. The FNM, LNP and MXP fuse the upper beak. The internal bony scaffolds of the upper beak are composed of the pnc and pmx, which are formed from the differentiation of mesenchyme cells derived from neural crest cells [64, 66]. The pmx is the most prominent functional and structural component of the upper beak, and its variations are often correlated with beak diversity [67]. During Hamburger and Hamilton (HH) [68] stages 28-30, the pmx forms and shapes while the pnc cease its expansion [9]. All embryos used in this study, thus, were matched with $\mathrm{HH} 28$ or $\mathrm{HH} 29$. We examined embryonic stages of the two tits based on the staging series of chicken, quail and some finches. Given that heterochrony of embryonic development exists between precocies and altrices [69], we referenced altricial embryonic days (ca. E6-6.5) corresponding to HH28/29 (see Additional file 1: Table S16).

\section{Embryo collection and tissue preparation}

We collected embryos during two breeding seasons (April 2013 to July 2013 and April 2014 to July 2014) in Nagqu, Tibet (Ground Tit) and Zuojia, Jilin (Great Tit) (see Additional file 1: Figure S4). In the breeding season, we observed and traced breeding pairs to locate nests and confirmed laying and hatching date according to their reproductive behaviors. Ground Tit females lay clutches of 4-9 eggs, and Great Tits lay 512 eggs, one per day [20]. Both species start their incubation upon laying their last eggs. We determined the initiation of the incubation according to behaviors of breeding pairs, such as feeding by males, staying time of females in nests. When these behaviors were 
observed, we dug nest burrows (Ground Tits) and examined nest boxes (Great Tits) to collect eggs and then incubated them in a micro-incubator at $38{ }^{\circ} \mathrm{C}$. At E6 and E6.5, we opened a few eggs to determine desired stages under stereoscope according to external identification features (see Additional file 1: Table S16); HH28 of Ground Tits matched with E6.5 and that of Great Tits with E6. We collected a total of 11 stage-matched Ground Tit embryos and 12 stagematched Great Tit embryos during two breeding seasons (see Additional file 1: Figure S5). Embryos were cleaned with cold $1 \times$ PBS and then stored in RNAhold (TranGen) at $4{ }^{\circ} \mathrm{C}$. Eight stage-matched Ground Tit and 9 Great Tit embryos were dissected for RNA extraction. Three matched embryos of each species were fixed in $4 \%$ paraformaldehyde (PFA) solution for in situ hybridization. The field collection of embryos was performed with the permission of the State Forestry Administration of China and conformed to the National Wildlife Conservation Law of China.

\section{Transcriptome sequencing, quality control and reads mapping}

Total RNA was extracted from four embryonic upper beaks from each species using TRIzol reagent (Invitrogen). The quality of extracted RNA samples was examined using a Nanodrop spectrophotometer and an Agilent 2100 Bioanalzyer. Sequencing libraries (non-strand-specific) were constructed for two qualified RNA samples from each species according to the manufacturer's protocol (Illumina). RNA sequencing was performed based on 100 bp paired-end reads using an Illumina HiSeq 2000 in the Novogene Bioinformatics Institute (Beijing, China), which produced a total of 21.91 GB raw reads. We used Cutadapt to remove adapters and reads with $>5 \%$ unidentified nucleotides [70], used FASTX-Toolkit (http://hannonlab.cshl.edu/fastx_toolkit) to remove reads with length of $<90$ and reads with $<90 \%$ bases that had Phred quality score $>20$, as well as used Trimmomatic to trim and pair reads using default setting [71]. The resulting 19.61 GB clean reads were retained for subsequent analyses: 21,689,370 and 22,209,206 reads for two Ground Tits and 28,570,866 and 25,599,330 reads for two Great Tits. An assessment for clean reads showed high quality with Q20 $>99.28 \%$ and Q30> 95.55\% (see Additional file 1: Table S17). The highquality reads of Ground Tits and Great Tits were mapped to respective genomes by TopHat 2.09 with default parameters [72].

\section{Gene quantification and differential expression analysis}

We quantified gene expression based on two data types; the simple read counts calculated by HTSeq-count [73], and the transformed TPM (transcripts per million) computed using a simple formula [74] from the measured FPKM (fragments per kilobase of exon model per million mapped reads) by Cufflinks [75]. The application of TPM was to take into account differences of library sizes between samples and the different length of genes between species. We identified 12,232 orthologous genes between the Ground Tit and the Great Tit. Genes were considered to be confidently expressed when their read counts $\geq 10$. But the genes with more than 2 -folds differences within species were removed. Final 9217 genes were retained for subsequent differential expression analysis. We compared gene expression patterns between Ground Tits and Great Tits using three different $\mathrm{R}$ programs, DESeq, edgeR and NOISeq. DESeq and edgeR were employed to input raw read counts, normalize counts with library size using their own methods, and perform differential expression analysis based on negative binomial distribution [76, 77]. Non-parametric NOISeq was applied to compare the TPM based on noise distribution that considers differences within species [78]. Genes were considered to be differentially expressed if they were observed to have a 2-folds change in expression and a FDR-adjusted $P$-value less than 0.001 [79]. To decrease false positives, we considered only genes that were identified by all three programs to have significantly differential expression with the same pattern (up-regulation or down-regulation). We used KOBAS [80] to enrich KEGG pathways and GO categories for these identified genes, with a focus on the pathways associated with the formation of cartilage and bone $[35,81]$.

\section{RT-qPCR and in situ hybridization}

To verify expression levels and patterns of genes identified by transcriptome analyses, we performed RT-qPCR for eight candidates in upper beaks of embryos because of limitation of embryo numbers (four replicates per species). Seven of them were selected from pathways related to bone development with the highest $P$-values and fold changes (Table 1). FRZB, although was not the one with the highest $P$-value and fold change, was annotated with a function in regulation of cartilage development (see Additional file 1: Table S12), therefore we also considered this gene for our following RT-qPCR. All primers were designed based on identical sequences of the relevant genes in both species (see Additional file 1: Table S18). Four isolated RNA samples from each species were converted to cDNA in a $50 \mu \mathrm{l}$ reaction using TransScript SuperMix (TranGen). cDNA, primers and TransStart SuperMix (TranGen) were mixed in a $20 \mu \mathrm{l}$ reaction to amplify each gene. RT-qPCR was run in triplicate on a Roche LightCycler 96 using a program: $94{ }^{\circ} \mathrm{C}$ 
for $30 \mathrm{~s}, 40$ cycles at $94{ }^{\circ} \mathrm{C}$ for $5 \mathrm{~s}, 60{ }^{\circ} \mathrm{C}$ for $15 \mathrm{~s}$ and $72{ }^{\circ} \mathrm{C}$ for $10 \mathrm{~s}$. The relative expression $(\Delta \mathrm{Cq})$ of all genes was normalized to glyceraldehyde-3phosphate dehydrogenase $(G A P D H)$, which is inversely proportional to the real expression levels. We examined significance in relative expression using Student's t-test. Expression differences were calculated using the $-\Delta \Delta \mathrm{Cq}$ method [82]. In situ hybridization was performed as previously described [83] on paraffin sections of embryonic heads at stage $\mathrm{HH} 28 / 29$ to assay spatial patterns of gene expression. Sections were hybridized with fluorescein-labeled RNA probes to FGF13, WIF1 and ITGB3 and hybridization signals were visualized using confocal epifluorescence.

\section{Functional test in chicken embryos}

Chicken embryos were manipulated using a biochemical strategy to test if changes in expression of the candidate genes, WIF1, FGF13 and ITGB3, can affect beak morphological traits. Eggs were incubated at $38{ }^{\circ} \mathrm{C}$ and 60 $70 \%$ humidity. At $\mathrm{HH} 30,10 \mu \mathrm{l}$ of recombinant mouse ITGB3 protein (200 ng/ $\mu \mathrm{l})$ (R\&D Systems) $(n=15)$, recombinant mouse WIF1 protein $(250 \mathrm{ng} / \mu \mathrm{l})(\mathrm{R} \& \mathrm{D}$ Systems) $(\mathrm{n}=15)$, and FGF13 recombinant protein (50 ng/ $\mu \mathrm{l})$ (Novus Biologicals) $(\mathrm{n}=15)$, was injected into the vitelline vein using a sterilized glass needle. Prior to injection, we opened an $\sim 2 \mathrm{~cm}$ oval window in minor diameter using a scalpel and removed the external membrane using fine forceps. Control embryos $(n=15)$ were treated with $0.1 \%$ bovine serum albumin (BSA). Surviving embryos were collected at $\mathrm{HH} 38$, fixed with $4 \%$ PFA and stored in $100 \%$ methanol after gradient dehydration. We collected a total of 43 survived embryos consisting of 13 controls, 12 rFGF13 treatments, 10 rITGB3 treatments, and 8 rWIF1 treatments.

Embryos were stained with $0.1 \%$ Alizarin Red, and cleared in gradient glycerol [84]. Lateral images of the embryonic specimens were captured on a stereo microscope. We defined upper and lower beak measurements in Image $(\mathrm{NIH})$ following Ealba's method [36]: upper beak measurement was taken from the tip of the nasal bone in the center of the maxilla to the distal tip of the premaxilla, and lower beak measurement was made from the proximal tip of the angular bone to the distal tip of the dentary bone (Fig. 4a). The values were presented as ratios of upper to lower beak measurements to eliminate individual and stage variations, and they were compared between treatments and controls by Student's t-test. To detect osteoblasts and osteoclasts in treatments separately, alkaline phosphatase (ALP) and tartrate-resistant acid phosphatase (TRAP) were stained in whole-mount embryos with Fast Red following Leukocyte Alkaline Phosphatase Kit and Acid Phosphatase Leukocyte kit (Sigma) protocols.

\section{Additional files}

Additional file 1: Supplementary materials. Included are 8 supplementary figures (Figures S1-S8) and 18 supplementary tables (Tables S1-S18). (PDF $1215 \mathrm{~kb})$

Additional file 2: The beak size data of 13 parids used in this study. (XLS 93 kb)

Additional file 3: The beak shape data of 13 parids used in this study. (XLS $91 \mathrm{~kb}$ )

Additional file 4: The description of expression abundance of the 623 DEGs. (XLS 217 kb)

\section{Abbreviations}

ALP: Alkaline phosphatase; BSA: Bovine serum albumin; CVA: Canonical variate analysis; FDR: False discovery rate; FNM: Frontal nasal mass; FPKM: Fragments per kilobase of exon model per million mapped reads; GO: Gene ontology; HH: Hamburger and Hamilton stage; KEGG: Kyoto encyclopedia of genes and genomes; LDA: Linear discriminant analysis; LNP: Lateral nasal prominence; MDP: Mandibular prominence; MXP: Maxillary prominence; PBS: Phosphate buffered saline; PFA: Paraformaldehyde; RTqPCR: Reverse transcription quantitative PCR; TPM: Transcripts per million; TRAP: Tartrate-resistant acid phosphatase

\section{Acknowledgments}

The authors thank Mingju E, Ye Gong and Changcao Wang for assisting in the sample collection, Qingsong Zhou for photographing the embryos, and Quan Kang and Xinhai Li for assistance with computer and statistical analysis. Many thanks to Herman Mays for discussion and comments on this manuscript. We sincerely thank the editor and reviewers for their valuable comments.

\section{Funding}

This work was supported by grants from the Strategic Priority Research Program of the Chinese Academy of Sciences (XDB13020300), the State Key Program of the NSFC (31330073) and the Ministry of Science and Technology of China (2014FY210200) to F.L.

\section{Availability of data and materials}

The morphological and expression data used in this study are available from Additional files. Sequencing data for the Ground Tit and the Great Tit have been deposited into the NCBI Sequence Read Archive (SRX760125SRX760128).

\section{Authors' contributions}

FL conceived the research program, FL, YQ, YC, BG and HW designed the experiments. SS and YC measured the specimens. YC, NH, BG, HW and XL collected embryonic samples. $Y C$ and $\mathrm{NH}$ performed the experiments. $Y C$, $B G, S W$ and $Y E Z$ analyzed the data and $Y C$ generated all figures. YC, BG, HW, SW, YEZ, YQ and FL wrote the initial manuscript. All authors contributed to writing the manuscript and approved the final manuscript.

\section{Ethics approval and consent to participate}

All animals in this study were maintained in accordance with guidelines established by the Institute of Zoology for the care and use of experimental animals. Our experiments were also approved by the animal experimental and medical ethics committee of the Institute of Zoology, Chinese Academy of Sciences.

\section{Competing interests}

The authors declare no competing financial interests.

\section{Publisher's Note}

Springer Nature remains neutral with regard to jurisdictional claims in published maps and institutional affiliations.

\section{Author details}

${ }^{1}$ Key Laboratory of Zoological Systematics and Evolution, Institute of Zoology, Chinese Academy of Sciences, Beijing 100101, China. ${ }^{2}$ School of Life 
Sciences, Northeast Normal University, Changchun 130024, China. ${ }^{3}$ School of Life Sciences, Jiangsu Normal University, Xuzhou 221116, China. ${ }^{4}$ University of Chinese Academy of Sciences, Beijing 100049, China. ${ }^{5}$ Department of Ecology, College of Life Sciences, Institute for Advanced Studies, Wuhan University, Wuhan 430072, China.

Received: 2 August 2017 Accepted: 28 November 2017

Published online: 21 December 2017

\section{References}

1. Grant PR, Grant BR. How and why species multiply: the radiation of Darwin's finches Princeton: University Press; 2011.

2. Patel NH. Evolutionary biology: how to build a longer beak. Nature. 2006;442:515-6.

3. Greenberg R, Cadena V, Danner RM, Tattersall GJ. Heat loss may explain bill size differences between birds occupying different habitats. PLoS One. 2012; 7:e40933.

4. Temeles EJ, Miller JS, Rifkin JL. Evolution of sexual dimorphism in bill size and shape of hermit hummingbirds (Phaethornithinae): a role for ecological causation. Philos Trans R Soc Lond Ser B Biol Sci. 2010;365:1053-63.

5. Carroll SB. Evo-devo and an expanding evolutionary synthesis: a genetic theory of morphological evolution. Cell. 2008;134:25-36.

6. Abzhanov A, Protas M, Grant BR, Grant PR, Tabin CJ. Bmp4 and morphological variation of beaks in Darwin's finches. Science. 2004;305: 1462-5.

7. Wu P, Jiang TX, Suksaweang S, Widelitz RB, Chuong CM. Molecular shaping of the beak. Science. 2004;305:1465-6.

8. Abzhanov A, Kuo WP, Hartmann C, Grant BR, Grant PR, Tabin CJ. The calmodulin pathway and evolution of elongated beak morphology in Darwin's finches. Nature. 2006:442:563-7.

9. Mallarino R, Campas O, Fritz JA, Burns KJ, Weeks OG, Brenner MP, Abzhanov A. Closely related bird species demonstrate flexibility between beak morphology and underlying developmental programs. Proc Natl Acad Sci U S A. 2012;109:16222-7.

10. Mallarino R, Grant PR, Grant BR, Herrel A, Kuo WP, Abzhanov A. Two developmental modules establish 3D beak-shape variation in Darwin's finches. Proc Natl Acad Sci U S A. 2011;108:4057-62.

11. Lamichhaney S, Berglund J, Almen MS, Maqbool K, Grabherr M, MartinezBarrio A, et al. Evolution of Darwin's finches and their beaks revealed by genome sequencing. Nature. 2015;518:371-5.

12. Lamichhaney S, Han F, Berglund J, Wang C, Almen MS, Webster MT, Grant BR, Grant PR, Andersson L. A beak size locus in Darwin's finches facilitated character displacement during a drought. Science. 2016;352:470-4

13. Fopplano S, Hu D, Marcucio RS. Signaling by bone morphogenetic proteins directs formation of an ectodermal signaling center that regulates craniofacial development. Dev Biol. 2007;312:103-14.

14. Song Y, Hui JN, Fu KK, Richman JM. Control of retinoic acid synthesis and FGF expression in the nasal pit is required to pattern the craniofacial skeleton. Dev Biol. 2004;276:313-29.

15. Minoux M, Rijli FM. Molecular mechanisms of cranial neural crest cell migration and patterning in craniofacial development. Development. 2010;137:2605-21.

16. Hume A. Stray notes on ornithology in India. Ibis. 1871;13:23-38.

17. Vaurie $C$. The birds of the Palearctic fauna: order Passeriformes. London: HF \& G. Witherby; 1959.

18. James HF, Ericson PGP, Slikas B, Lei FM, Gill FB, Olson SL. Pseudopodoces humilis, a misclassified terrestrial tit (Paridae) of the Tibetan plateau: evolutionary consequences of shifting adaptive zones. Ibis. 2003;145:185-202

19. Qu Y, Zhao H, Han N, Zhou G, Song G, Gao B, et al. Ground tit genome reveals avian adaptation to living at high altitudes in the Tibetan plateau. Nat Commun. 2013;4:2071.

20. del Hoyo J, Elliott A, Sargatal J, Christie DA, de Juana E. Handbook of the birds of the world alive. Barcelona: Lynx Edicions; 2014

21. Ke DH, Lu X. Burrow use by Tibetan Ground Tits Pseudopodoces humilis: coping with life at high altitudes. Ibis. 2009;151:321-31.

22. Lu X, Yu TL, Ke DH. Helped ground tit parents in poor foraging environments reduce provisioning effort despite nestling starvation. Anim Behav. 2011;82:861-7.
23. Shao S, Quan Q, Cai T, Song G, Qu Y, Lei F. Evolution of body morphology and beak shape revealed by a morphometric analysis of 14 Paridae species. Front Zool. 2016;13:30.

24. Laine VN, Gossmann TI, Schachtschneider KM, Garroway CJ, Madsen O, Verhoeven KJF, et al. Evolutionary signals of selection on cognition from the great tit genome and methylome. Nat Commun. 2016;7:10474.

25. Pires-daSilva A, Sommer RJ. The evolution of signalling pathways in animal development. Nat Rev Genet. 2003;4:39-49.

26. Favre A, Packert M, Pauls SU, Jahnig SC, Uhl D, Michalak I, Muellner-Riehl AN. The role of the uplift of the Qinghai-Tibetan plateau for the evolution of Tibetan biotas. Biol Rev Camb Philos Soc. 2015;90:236-53.

27. Carroll SB. Evolution at two levels: on genes and form. PLoS Biol. 2005;3: 1159-66.

28. Abzhanov A, Tabin CJ. Shh and Fgf8 act synergistically to drive cartilage outgrowth during cranial development. Dev Biol. 2004;273:134-48.

29. Bhullar BA, Morris ZS, Sefton EM, Tok A, Tokita M, Namkoong B, Camacho J, Burnham DA, Abzhanov A. A molecular mechanism for the origin of a key evolutionary innovation, the bird beak and palate, revealed by an integrative approach to major transitions in vertebrate history. Evolution. 2015;69:1665-77.

30. Medio M, Yeh E, Popelut A, Babajko S, Berdal A, Helms JA. Wnt/beta-catenin signaling and Msx1 promote outgrowth of the maxillary prominences. Front Physiol. 2012;3:375.

31. Soneson C, Delorenzi M. A comparison of methods for differential expression analysis of RNA-seq data. BMC Bioinformatics. 2013;14:91.

32. Hu D, Marcucio RS, Helms JA. A zone of frontonasal ectoderm regulates patterning and growth in the face. Development. 2003;130:1749-58.

33. Schneider RA, Helms JA. The cellular and molecular origins of beak morphology. Science. 2003;299:565-8.

34. Marie PJ. Fibroblast growth factor signaling controlling osteoblast differentiation. Gene. 2003;316:23-32.

35. Hall J, Jheon AH, Ealba EL, Eames BF, Butcher KD, Mak SS, Ladher R, Alliston T, Schneider RA. Evolution of a developmental mechanism: species-specific regulation of the cell cycle and the timing of events during craniofacial osteogenesis. Dev Biol. 2014;385:380-95.

36. Ealba EL, Jheon AH, Hall J, Curantz C, Butcher KD, Schneider RA. Neural crest-mediated bone resorption is a determinant of species-specific jaw length. Dev Biol. 2015;408:151-63.

37. McHugh KP, Hodivala-Dilke K, Zheng MH, Namba N, Lam J, Novack D, et al. Mice lacking beta 3 integrins are osteosclerotic because of dysfunctional osteoclasts. J Clin Invest. 2000;105:433-40.

38. Purdue PE, Crotti TN, Shen ZX, Swantek J, Li J, Hill J, et al. Comprehensive profiling analysis of actively resorbing osteoclasts identifies critical signaling pathways regulated by bone substrate. Sci Rep. 2014;4:7595.

39. Zhao HB, Kitaura H, Sands MS, Ross FP, Teitelbaum SL, Novack DV. Critical role of beta 3 integrin in experimental postmenopausal osteoporosis. J Bone Miner Res. 2005:20:2116-23.

40. Simonet WS, Lacey DL, Dunstan CR, Kelley M, Chang MS, Luthy R, et al. Osteoprotegerin: a novel secreted protein involved in the regulation of bone density. Cell. 1997;89:309-19.

41. Richman JM, Lee SH. About face: signals and genes controlling jaw patterning and identity in vertebrates. BioEssays. 2003;25:554-68.

42. Herrel A, Podos J, Huber SK, Hendry AP. Bite performance and morphology in a population of Darwin's finches: implications for the evolution of beak shape. Funct Ecol. 2005;19:43-8.

43. Soons J, Genbrugge A, Podos J, Adriaens D, Aerts P, Dirckx J, Herrel A. Is beak morphology in Darwin's finches tuned to loading demands? PLoS One. 2015;10:e0129479.

44. Amadon D. Bird weights as an aid in taxonomy. Wilson Bull. 1943;55:164-77.

45. Blomberg SP, Garland T, Ives AR. Testing for phylogenetic signal in comparative data: behavioral traits are more labile. Evolution. 2003;57: 717-45.

46. Pagel M. Inferring the historical patterns of biological evolution. Nature. 1999:401:877-84.

47. Johansson US, Ekman J, Bowie RC, Halvarsson P, Ohlson J, Price TD, Ericson PG. A complete multilocus species phylogeny of the tits and chickadees (Aves: Paridae). Mol Phylogenet Evol. 2013;69:852-60.

48. Zelditch M, Swiderski D, Sheets H, Fink W. Geometric morphometrics for biologists: a primer. London: Elsevier Academic Press; 2004.

49. Rohlf FJ. Shape statistics: procrustes superimpositions and tangent spaces. Classif. 1999;16:197-223. 
50. Klingenberg CP, Gidaszewski NA. Testing and quantifying phylogenetic signals and homoplasy in morphometric data. Syst Biol. 2010;59:245-61.

51. Klingenberg CP. MorphoJ: an integrated software package for geometric morphometrics. Mol Ecol Resour. 2011;11:353-7.

52. Klingenberg CP, Marugan-Lobon J. Evolutionary covariation in geometric morphometric data: analyzing integration, modularity, and allometry in a phylogenetic context. Syst Biol. 2013;62:591-610.

53. Campbell NA, Atchley WR. The geometry of canonical variate analysis. Syst Zool. 1981;30:268-80

54. Timm NH. Applied multivariate analysis. New York: Springer; 2002.

55. Paradis E, Claude J, Strimmer K. APE: analyses of phylogenetics and evolution in R languag. Bioinformatics. 2004;20:289-90.

56. Maddison WP. Squared-change parsimony reconstructions of ancestral states for continuous-valued characters on a phylogenetic tree. Syst Zool. 1991:40:304-14.

57. Zhang GJ, Li C, Li QY, Li B, Larkin DM, Lee C, et al. Comparative genomics reveals insights into avian genome evolution and adaptation. Science. 2014; 346:1311-20.

58. Bouckaert R, Heled J, Kuhnert D, Vaughan T, Wu CH, Xie D, Suchard MA Rambaut A, Drummond AJ. BEAST 2: a software platform for Bayesian evolutionary analysis. PLoS Comput Biol. 2014;10:e1003537.

59. Yang ZH. PAML 4: Phylogenetic analysis by maximum likelihood. Mol Biol Evol. 2007;24:1586-91.

60. Goodman M, Sterner KN, Islam M, Uddin M, Sherwood CC, Hof PR, et al. Phylogenomic analyses reveal convergent patterns of adaptive evolution in elephant and human ancestries. Proc Natl Acad Sci U S A. 2009;106:20824-9.

61. Yang ZH. Likelihood ratio tests for detecting positive selection and application to primate lysozyme evolution. Mol Biol Evol. 1998;15:568-73.

62. Storey JD, Tibshirani R. Statistical significance for genomewide studies. Proc Natl Acad Sci U S A. 2003;100:9440-5.

63. Lumsden A, Sprawson N, Graham A. Segmental origin and migration of neural crest cells in the hindbrain region of the chick embryo. Development. 1991;113:1281-91.

64. Kontges G, Lumsden A. Rhombencephalic neural crest segmentation is preserved throughout craniofacial ontogeny. Development. 1996;122: 3229-42.

65. Francis-West $P$, Ladher R, Barlow A, Graveson A. Signalling interactions during facial development. Mech Dev. 1998;75:3-28.

66. Helms JA, Schneider RA. Cranial skeletal biology. Nature. 2003:423:326-31.

67. Hanken J, Hall BK. The skull. Chicago: University of Chicago Press; 1993.

68. Hamburger $\mathrm{V}$, Hamilton $\mathrm{HL}$. A series of normal stages in the development of the chick embryo. J Morphol. 1951;88:49-92.

69. Blom J, Lilja C. A comparative study of embryonic development of some bird species with different patterns of postnatal growth. Zoology (Jena). 2005;108:81-95

70. Martin M. Cutadapt removes adapter sequences from high-throughput sequencing reads. Embnet Journal. 2011;17:10-2.

71. Bolger AM, Lohse M, Usadel B. Trimmomatic: a flexible trimmer for Illumina sequence data. Bioinformatics. 2014;30:2114-20.

72. Kim D, Pertea G, Trapnell C, Pimentel H, Kelley R, Salzberg SL. TopHat2: accurate alignment of transcriptomes in the presence of insertions, deletions and gene fusions. Genome Biol. 2013;14:R36.

73. Anders S, Pyl PT, Huber W. HTSeq-a python framework to work with highthroughput sequencing data. Bioinformatics. 2015:31:166-9.

74. Pachter L. Models for transcript quantification from RNA-Seq. 2011. http:// arxiv.org/abs/1104.3889. Accessed 10 Oct 2017

75. Trapnell C, Williams BA, Pertea G, Mortazavi A, Kwan G, van Baren MJ, Salzberg SL, Wold BJ, Pachter L. Transcript assembly and quantification by RNA-Seq reveals unannotated transcripts and isoform switching during cell differentiation. Nat Biotechnol. 2010;28:511-5.

76. Anders S, Huber W. Differential expression analysis for sequence count data. Genome Biol. 2010;11:R106

77. Robinson MD, McCarthy DJ, Smyth GK. edgeR: a bioconductor package for differential expression analysis of digital gene expression data. Bioinformatics. 2010;26:139-40.

78. Tarazona S, Garcia-Alcalde F, Dopazo J, Ferrer A, Conesa A. Differential expression in RNA-seq: a matter of depth. Genome Res. 2011;21:2213-23.

79. Schartl M, Kneitz S, Wilde B, Wagner T, Henkel CV, Spaink HP, Meierjohann S. Conserved expression signatures between medaka and human pigment cell tumors. PLoS One. 2012;7:e37880.
80. Xie C, Mao X, Huang J, Ding Y, Wu J, Dong S, et al. KOBAS 2.0: a web server for annotation and identification of enriched pathways and diseases. Nucleic Acids Res. 2011;39:316-22.

81. Eames BF, Schneider RA. The genesis of cartilage size and shape during development and evolution. Development. 2008;135:3947-58.

82. Livak KJ, Schmittgen TD. Analysis of relative gene expression data using real-time quantitative PCR and the $2(T)(-$ Delta Delta C) method. Methods. 2001;25:402-8.

83. Abzhanov A. In situ hybridization analysis of embryonic beak tissue from Darwin's finches. Cold Spring Harb Protoc. 2009;4:pdb-prot5175.

84. Wassersug RJ. A procedure for differential staining of cartilage and bone in whole formalin-fixed vertebrates. Stain Technol. 1976;51:131-4.

\section{Submit your next manuscript to BioMed Central and we will help you at every step:}

- We accept pre-submission inquiries

- Our selector tool helps you to find the most relevant journal

- We provide round the clock customer support

- Convenient online submission

- Thorough peer review

- Inclusion in PubMed and all major indexing services

- Maximum visibility for your research

Submit your manuscript at www.biomedcentral.com/submit
) Biomed Central 